Article

\title{
Rhodanineacetic Acid Derivatives as Potential Drugs: Preparation, Hydrophobic Properties and Antifungal Activity of (5-Arylalkylidene-4-oxo-2-thioxo-1,3-thiazolidin-3-yl)acetic Acids
}

Jan Dolezel ${ }^{1}$, Petra Hirsova ${ }^{1}$, Veronika Opletalova ${ }^{1}$, Jiri Dohnal ${ }^{2,3}$, Vejsova Marcela ${ }^{4}$, Jiri Kunes 5 and Josef Jampilek ${ }^{2,3, *}$

1 Department of Pharmaceutical Chemistry and Drug Control, Faculty of Pharmacy in Hradec Kralove, Charles University in Prague, Heyrovskeho 1203, 50005 Hradec Kralove, Czech Republic; E-Mails: veronika.opletalova@faf.cuni.cz (V.O.); jan.dolezel@faf.cuni.cz (J.D.); hirsovap@1fhk.cuni.cz (P.H.)

2 Zentiva k.s., U kabelovny 130, 10237 Prague 10, Czech Republic;

E-Mail: jiri.dohnal@zentiva.cz (J.D.)

3 Department of Chemical Drugs, Faculty of Pharmacy, University of Veterinary and Pharmaceutical Sciences, Palackeho 1-3, 61242 Brno, Czech Republic

4 Department of Clinical Microbiology, Charles University Medical School and Teaching Hospital, Sokolska 581, Hradec Kralove 500 05, Czech Republic;

E-Mail: marcela.vejsova@faf.cuni.cz (M.V.)

5 Department of Inorganic and Organic Chemistry, Faculty of Pharmacy in Hradec Kralove, Charles University in Prague, Heyrovskeho 1203, 50005 Hradec Kralove, Czech Republic; E-Mail: jiri.kunes@faf.cuni.cz (J.K.)

* Author to whom correspondence should be addressed; E-Mail: josef.jampilek@zentiva.cz; Tel.: +420267243695; Fax: +420272701331.

$\dagger$ Preliminary results connected with the topic of the article were presented at The Twelfth Electronic Conference on Synthetic Organic Chemistry (ECSOC-12, http://www.usc.es/congresos/ecsoc/12/ ECSOC12.htm), November 1-30, 2008, paper C0006.

Received: 21 September 2009; in revised form: 9 October 2009/ Accepted: 19 October 2009 / Published: 20 October 2009

Abstract: Some [(5Z)-(5-arylalkylidene-4-oxo-2-thioxo-1,3-thiazolidin-3-yl)]acetic acids were prepared as potential antifungal compounds. The general synthetic approach to all 
synthesized compounds is presented. Lipophilicity of all the discussed rhodanine-3-acetic acid derivatives was analyzed using a reversed phase high performance liquid chromatography (RP-HPLC) method. The procedure was performed under isocratic conditions with methanol as an organic modifier in the mobile phase using an end-capped non-polar $\mathrm{C}_{18}$ stationary RP column. The RP-HPLC retention parameter $\log k$ (the $\operatorname{logarithm}$ of the capacity factor $k$ ) is compared with $\log P$ values calculated in silico. All compounds were evaluated for antifungal effects against selected fungal species. Most compounds exhibited no interesting activity, and only \{(5Z)-[4-oxo-5-(pyridin-2ylmethylidene)-2-thioxo-1,3-thiazolidin-3-yl] \}acetic acid strongly inhibited the growth of Candida tropicalis 156, Candida krusei E 28, Candida glabrata 20/I and Trichosporon asahii 1188.

Keywords: [(5Z)-(5-arylalkylidene-4-oxo-2-thioxo-1,3-thiazolidin-3-yl)]acetic acids; synthesis; lipophilicity measurement; in vitro antifungal activity

\section{Introduction}

In 1997, a study based on a database search showed that the prevalence of rhodanine-containing compounds of pharmaceutical interest is very small, despite the fact that the compounds exhibit a wide variety of bioactivities [1]. One of the reasons may be poor solubility of rhodanine derivatives in water, but in rhodanine-3-acetic acids this problem can be overcome by preparing suitable salts.

Rhodanine-3-acetic acid (RAA) was prepared by Körner [2] in 1908, and condensation products of the acid with various aldehydes were reported in the same year [3]. Since that time, many (5-arylalkylidene-4-oxo-2-thioxo-1,3-thiazolidin-3-yl)alkanoic acids have been prepared and studied as potential antimycobacterial [4,5], antifungal [6-15], pesticidal [16-18], antihypertensive [19], and antineoplastic [20,21] agents. Rhodanine carboxylic acid derivatives have also be patented for the treatment and prevention of metabolic bone disorders. It was found that they stimulate parathyroid hormone receptor-mediated cAMP formation and could be useful for the local and systemic treatment of rheumatoid arthritis, osteoarthritis and degenerative arthrosis [22,23]. Trypanocidal activity of substituted rhodanine-3-acetic acids has been reported recently [24]. The only rhodanineacetic acid derivative that has been used clinically is the aldose reductase inhibitor epalrestat (Figure 1). It is marketed in Japan and used to slow eye damage associated with diabetes and to prevent diabetic peripheral neuropathy [1,25-28]. Aldose reductase is not the only enzyme inhibited by rhodaninecarboxylic acids. It was found that many other enzymes are inhibited by the derivatives of this structural class, and the enzyme inhibition may be, at least in part, the mechanism responsible for various biological effects of rhodanine derivatives [29]. 
Figure 1. Structure of epalrestat.

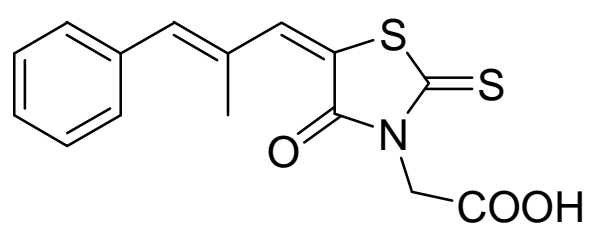

Determination of the physico-chemical parameters of biologically active compounds has become more important in an age of rational thinking in drug design [30]. One of the major prerequisites for pharmacological screening and drug development is prediction of absorption, e.g., transport of a molecule through cellular membranes, i.e. bioavailability. Most frequently, the drugs cross biological barriers by passive transport, which strongly depends on lipophilicity. Therefore hydrophobicity is one of the most important physical properties of biologically active compounds. This thermodynamic parameter describes the partitioning of a compound between an aqueous and an organic phase and is characterized by the partition coefficient $P$ [31,32]. For practical purposes, partition coefficient is mostly used in its logarithmic form $\log P$. Classical methods for determination of this constant are time consuming and not always sufficiently reliable. Therefore, reversed phase high performance liquid chromatography (RP-HPLC) methods have become popular and are widely used for lipophilicity measurement [33]. This paper is a follow-up work to previous papers [34-46] aimed at the synthesis, physicochemical properties and biological testing of newly prepared potential drugs based on nitrogen containing heterocycles.

\section{Results and Discussion}

\subsection{Chemistry}

The preparation of the studied compounds is indicated in Scheme 1. The purity of samples was checked by elemental analysis. Their structures were confirmed by melting points and spectral data (UV, IR, ${ }^{1} \mathrm{H}$ - and $\left.{ }^{13} \mathrm{C}-\mathrm{NMR}\right)$. Compounds 1-4 have been reported previously. [(5Z)-(5-Benzylidene-4oxo-2-thioxo-1,3-thiazolidin-3-yl)]acetic acid (1) has been known since 1908 [3], but its NMR characterization was only performed as late as 1982 [27]. In 2006, the compound was prepared under microwave irradiation [47]. The condensation products of rhodanine-3-acetic acid with pyridinecarbaldehydes were prepared in 1961 as potential antibacterial and antifungal agents [8]. $\{(5 Z)$-[4-Oxo-5-(pyridin-2-ylmethylidene)-2-thioxo-1,3-thiazolidin-3-yl] $\}$ acetic acid (2) was patented as a potential drug for the treatment of metabolic bone diseases [22,23]. The ${ }^{1} \mathrm{H}-\mathrm{NMR}$ spectra of $\mathbf{3}$ and 4 were published by Tanaouchi et al. [27,28]. The remaining products are novel compounds.

Arylalkylidenerhodanines can form two isomers. According to references [48-51], syntheses of these compounds result in the thermodynamically more stable Z-izomers. Configuration on the exocyclic double bond can be determined on the basis of NMR spectra. ${ }^{1} \mathrm{H}-\mathrm{NMR}$ signals of the methine-group hydrogens for Z-isomers are more downfield compared to E-isomers. Calculated and previously reported experimental values of ${ }^{1} \mathrm{H}-\mathrm{NMR}$ shifts for the products reported in the present paper are given in Table 1. Based on our experimental NMR results the compounds presented here 
were obtained as single isomers. Their shifts for methine proton range between 7.86-7.94 ppm. Hence, it can be concluded that they are $Z$-isomers.

Scheme 1. Synthesis and structures of the target 5-substituted rhodanine-3-acetic acid derivatives 1-9.
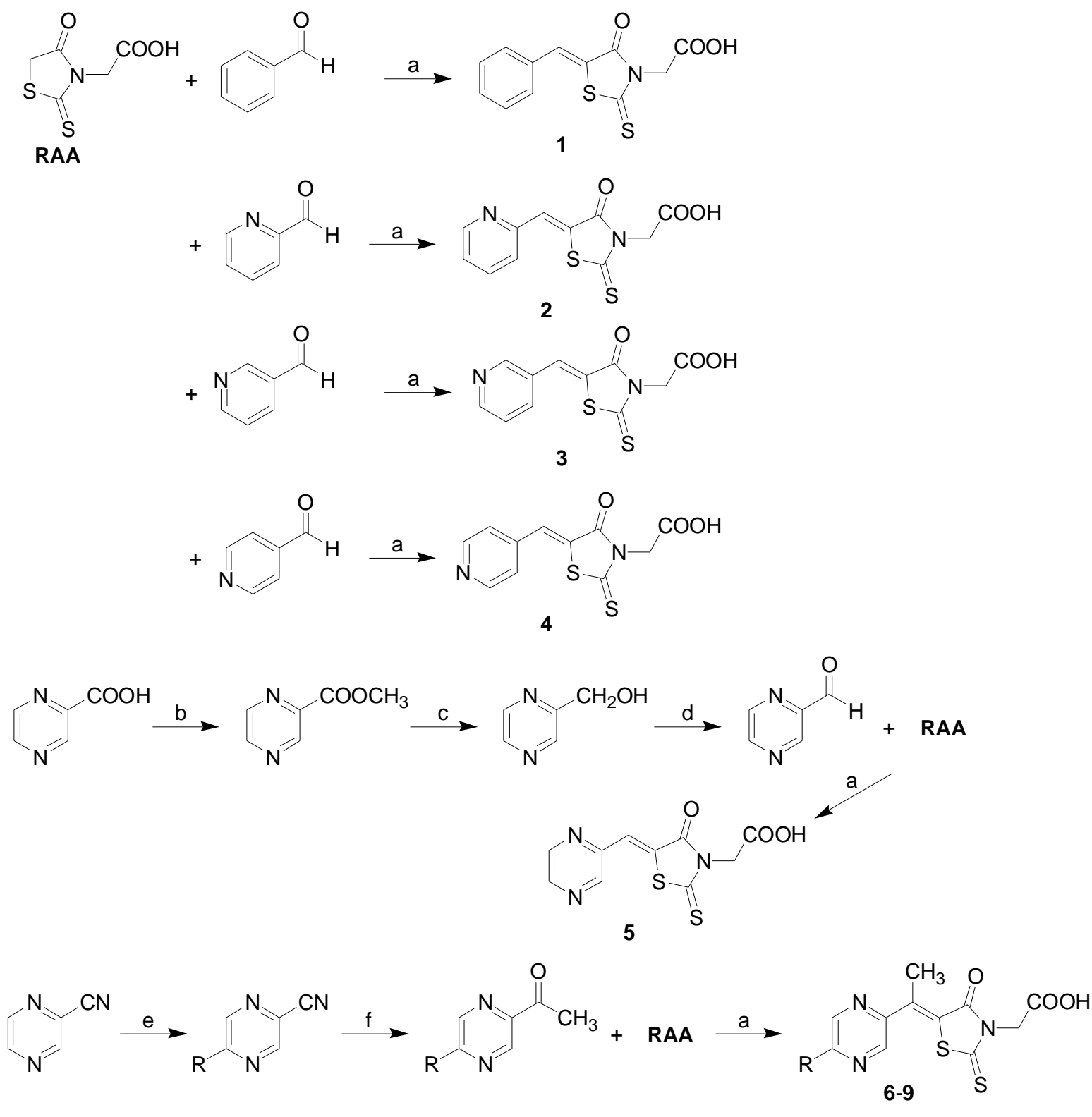

$\mathrm{R}: \mathbf{6}=\mathrm{H} ; \mathbf{7}=$ butyl; 8 = tert-butyl; $\mathbf{9}=$ benzyl

Reagents and Conditions: a) $\mathrm{CH}_{3} \mathrm{COOH}, \mathrm{CH}_{3} \mathrm{COONa},\left(\mathrm{CH}_{3} \mathrm{CO}\right)_{2} \mathrm{O}$; b) $\mathrm{MeOH}, \mathrm{H}_{2} \mathrm{SO}_{4}$; c) $\mathrm{NaBH}_{4}$, $\mathrm{H}_{2} \mathrm{O}$; d) $\mathrm{MnO}_{2}$, acetone, sonification; e) R-COOH, $\mathrm{AgNO}_{3},\left(\mathrm{NH}_{4}\right)_{2} \mathrm{~S}_{2} \mathrm{O}_{8}, \mathrm{H}_{2} \mathrm{O}$; f) $\mathrm{CH}_{3} \mathrm{MgI}_{2} \mathrm{Et}_{2} \mathrm{O}$.

\subsection{Lipophilicity}

Many low molecular weight drugs cross biological membranes through passive transport, which strongly depends on their lipophilicity. Lipophilicity is a property that has a major effect on absorption, distribution, metabolism, excretion, and toxicity (ADME/Tox) properties as well as 
pharmacological activity. Lipophilicity has been studied and applied as an important drug property for decades [52].

$\log P$ is the logarithm of the partition coefficient in a biphasic system (e.g., $n$-octanol/water), defined as the ratio of compound concentration in both organic/inorganic phases. The $\log P$ is determined for uncharged species of the drug. Clog $P$ values present the logarithm of $n$-octanol/water partition coefficient based on established chemical interactions.

Table 1. Lipophilicities and ${ }^{1} \mathrm{H}-\mathrm{NMR}$ shifts for the methine hydrogen $(\delta$, ppm) of 5-arylalkylidene-3-carboxymethylrhodanines 1-9.

\begin{tabular}{|c|c|c|c|c|c|c|}
\hline & & & $\left.\right|_{S} \int^{O}-C$ & & & \\
\hline Comp. & $\mathbf{R}$ & $\log k$ & $\log P$ & $Z$ & $\boldsymbol{E}$ & ND \\
\hline 1 & & 0.2013 & $\begin{array}{c}2.34 \pm 0.81^{a} \\
1.54^{b}\end{array}$ & $7.41^{b}$ & $6.80^{b}$ & $\begin{array}{l}7.88^{c} \\
7.81^{d}\end{array}$ \\
\hline 2 & & 0.1399 & $\begin{array}{c}0.85 \pm 0.82^{a} \\
0.62^{b}\end{array}$ & $7.62^{b}$ & $7.01^{b}$ & - \\
\hline 3 & & 0.1116 & $\begin{array}{c}1.10 \pm 0.82^{a} \\
0.20^{b}\end{array}$ & $7.41^{b}$ & $6.80^{b}$ & $7.95^{c}$ \\
\hline 4 & & 0.1342 & $\begin{array}{c}0.85 \pm 0.82^{a} \\
0.20^{b}\end{array}$ & $7.39^{b}$ & $6.78^{b}$ & $7.87^{c}$ \\
\hline 5 & & 0.1165 & $\begin{array}{c}0.09 \pm 0.82^{a} \\
-0.71^{b}\end{array}$ & $7.41^{b}$ & $6.80^{b}$ & - \\
\hline 6 & & 0.1734 & $\begin{array}{c}0.65 \pm 0.84^{a} \\
-0.54^{b}\end{array}$ & - & - & - \\
\hline 7 & & 0.2270 & $\begin{array}{c}2.70 \pm 0.84^{a} \\
1.49^{b}\end{array}$ & - & - & - \\
\hline 8 & & 0.2301 & $\begin{array}{c}2.33 \pm 0.84^{a} \\
1.59^{b}\end{array}$ & - & - & - \\
\hline 9 & & 0.2276 & $\begin{array}{c}2.64 \pm 0.84^{a} \\
1.84^{b}\end{array}$ & - & - & - \\
\hline
\end{tabular}

${ }^{a} \mathrm{ACD} / \mathrm{LogP}$, ver. $1.0 ;{ }^{b} \mathrm{CS}$ ChemOffice Ultra, ver. 7.0; ${ }^{c} \mathrm{DMSO}-d_{6}$, ref. [27,28]; ${ }^{d} \mathrm{DMSO}-d_{6}$, ref. [47]; $\mathrm{ND}=$ not defined.

It was recognised that the retention of a compound in the reversed-phase liquid chromatography is governed by its lipophilicity, and thus shows correlation with the octanol-water partition coefficient [53]. High performance liquid chromatography (HPLC) provides an excellent platform for computer controlled automated measurements with computerised data acquisition for a large number of 
compounds. Other advantages in the use of HPLC retention data for lipophilicity determination are the absence of need for concentration determination and method validation, separation of small impurities from the main component, sufficiency of small amounts of material for measurements and possibility of their full automation. Therefore, the investigation of the true potential of this method is of great importance [54].

An excellent review of the effect of stationary and mobile phase selection has been published by van der Waterbeemd et al. [32] and more recently by Claessens et al. [55]. RP-HPLC methods have become popular and widely used for lipophilicity measurements. A general procedure is the measurement of the directly accessible retention time under isocratic conditions with varying amounts of methanol as an organic modifier in the mobile phase using end-capped non-polar $\mathrm{C}_{18}$ stationary RP columns and calculating capacity factors $k$. Log $k$ is the logarithm of capacity factors in chromatographic approaches, which is related to the partitioning of a compound between a mobile and a (pseudo-)stationary phase. $\log k$ is used as the lipophilicity index converted to $\log P$ scale $[32,33$, 54-57].

Some groups used a $\mathrm{C}_{18}$ chromatographic column with methanol-water mobile phases to obtain $\log k_{\mathrm{w}}$, i.e., $\log k$ extrapolated to $0 \%$ organic modifier, as an alternative to $\log P$ [58]. The $\log k_{\mathrm{w}}$ is obtained by performing several measurements with various ratios of water/organic solvent. Nevertheless, determination of $\log k_{\mathrm{w}}$ has some disadvantages. Its determination is time consuming due to a number of measurements before the calculation of $\log k_{\mathrm{w}}$ [53]. Many studies [59-61] showed that for heteroaromatic compounds in which various intramolecular interactions between heteroatoms and substituents occur, it is more convenient to determine log $k$ using mobile phases containing around $50 \%$ methanol. Therefore, this study was performed using methanol/water (70:30) as the mobile phase. The conditions (non-buffered mobile phase) were chosen with respect to conditions of biological evaluations, which are performed mostly under neutral conditions $(\mathrm{pH} \sim 7)$.

Hydrophobicities $(\log P)$ of the studied compounds 1-9 were calculated using ACD/LogP, version 1.0 (Advanced Chemistry Development Inc., Toronto, ON, Canada) and CS ChemOffice Ultra, version 7.0 (CambridgeSoft, Cambridge MA, U.S.A.) and measured by means of RP-HPLC determination of capacity factors $k$ with a subsequent calculation of $\log k$. The results are summarized in Table 1 and illustrated in Figure 2.

As expected, methylation of the connection linker increases lipophilicity, i.e., compound $\mathbf{5}$ $(\log k=0.1165)$ is less hydrophobic than its alkylated derivative $6(\log k=0.1734)$. Among the arylmethylidene derivatives [(5Z)-(5-benzylidene-4-oxo-2-thioxo-1,3-thiazolidin-3-yl)]acetic acid (1) is more lipophilic than its 5-heteroarymethylidene congeners 2-5. [(5Z)-\{5-[1-(5-tert-Butylpyrazin-2yl)ethylidene]-4-oxo-2-thioxo-1,3-thiazolidin-3-yl\}] acetic acid (8) is the most lipophilic compound, which is in a good agreement with the results of our previous studies [36-38,43,44].

\subsection{In vitro antifungal activity}

Antifungal activity of all compounds against Candida albicans ATCC 44859 (CA), Candida tropicalis 156 (CT), Candida krusei E 28 (CK), Candida glabrata 20/I (CG), Trichosporon asahii 1188 (TA), Aspergillus fumigatus 231 (AF), Absidia corymbifera 272 (AC) and Trichophyton mentagrophytes 445 (TM) was evaluated by the microdilution broth method. These clinical isolates 
were obtained from the Department of Clinical Microbiology,University Hospital and Faculty of Medicine, Charles University, Prague, Czech Republic. Solubility of all compounds for biological experiments was satisfactory, and the preparation of suitable salts was not necessary. The prepared substances did not show antifungal activity, except for \{(5Z)-[4-oxo-5-(pyridin-2-ylmethylidene)-2thioxo-1,3-thiazolidin-3-yl] \}acetic acid (2), which strongly inhibited the growth of Candida tropicalis 156, Candida krusei E 28, Candida glabrata 20/I and Trichosporon asahii 1188. This is rather surprising since Sortino and co-workers [62], who studied antifungal properties of 3-unsubstituted 5 -arylalkylidenerhodanines, found that the replacement of benzene ring with a pyridine ring resulted in the loss of antifungal activity. Antifungal properties of [(5Z)-(5-pyrazin-2-ylalkylidene-4-oxo-2thioxo-1,3-thiazolidin-3-yl)]acetic acids 5-9 have not been studied so far. Similarly to 3-unsubstituted (5Z)-5-pyrazine-2-ylalkylidene-2-thioxo-1,3-thiazolidin-4-ones, that have also been prepared and tested in our laboratory $[43,63]$, their corresponding 3-carboxymethyl congeners reported here were also inactive.

Figure 2. Comparison of the $\log P$ data calculated using the two programs with the experimentally found $\log k$ values. The compounds are arranged in the ascending manner according to the experimental $\log k$ values.

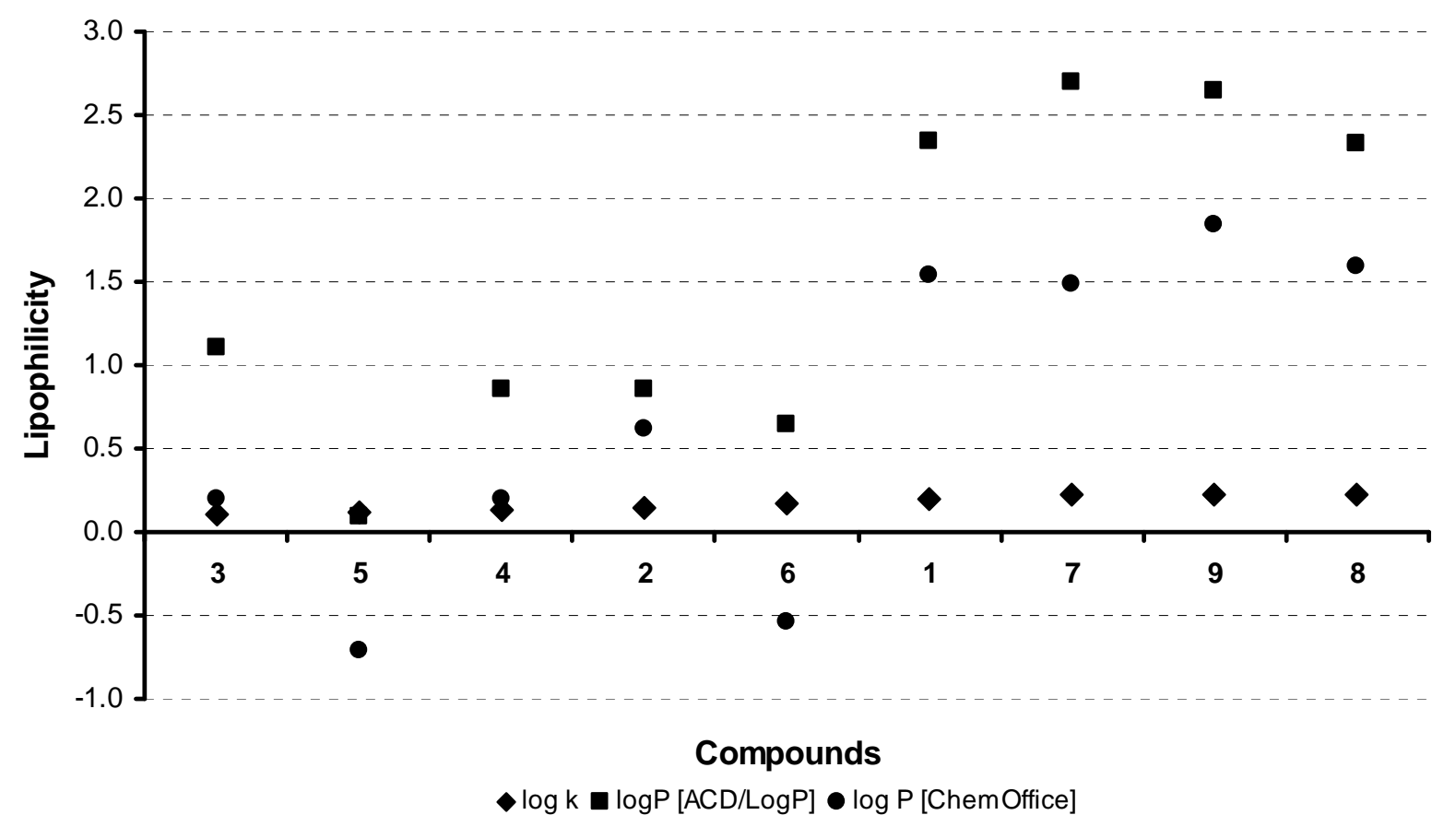

Little is known about the molecular mechanisms responsible for the antifungal properties of rhodanine-3-acetic derivatives. Orchard et al. [13] found that phenylalkoxy substituted (5-benzylidene4-oxo-2-thioxo-1,3-thiazolidin-3-yl)acetic acids, for example compound 10 (Figure 3), inhibit fungal protein mannosyl transferase 1 (PMT1) and Candida albicans proliferation in vitro. In some cases, inhibitory activity against the organism outstripped that versus isolated enzyme (PMT1). This indicates an off-target activity (including inhibition of other PMT subtypes) contributing to the anti-Candida effects, or increased penetration to the endoplasmic reticulum membrane. 
Figure 3. Structure of compound 10, PMT1 inhibitor.

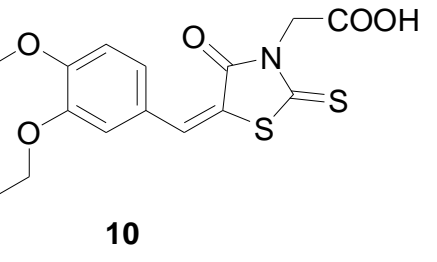

Table 2. In vitro antifungal activity $\left(\mathrm{IC}_{80}\right)$ of compounds $\mathbf{1 - 9}$ compared with fluconazole (FLU) standard.

\begin{tabular}{|c|c|c|c|c|c|c|c|c|c|c|c|}
\hline \multirow{2}{*}{\multicolumn{2}{|c|}{ Strain }} & \multicolumn{10}{|c|}{$\mathrm{MIC} / \mathrm{IC}_{80}[\mu \mathrm{mol} / \mathrm{L}]$} \\
\hline & & 1 & 2 & 3 & 4 & 5 & 6 & 7 & 8 & 9 & FLU \\
\hline \multirow{2}{*}{$\mathbf{C A}$} & $24 h$ & $>500$ & 500 & $>500$ & $>500$ & $>250$ & $>500$ & $>500$ & $>500$ & 500 & 1.09 \\
\hline & $48 h$ & $>500$ & 500 & $>500$ & $>500$ & $>250$ & $>500$ & $>500$ & $>500$ & $>500$ & 2.17 \\
\hline \multirow{2}{*}{ CT } & $24 h$ & $>500$ & 0.98 & 500 & $>500$ & $>250$ & 500 & 500 & $>500$ & $>500$ & 2,72 \\
\hline & $48 h$ & $>500$ & 1.95 & $>500$ & $>500$ & $>250$ & $>500$ & $>500$ & $>500$ & $>500$ & 5.44 \\
\hline \multirow{2}{*}{$\mathbf{C K}$} & $24 h$ & $>500$ & 1.95 & 500 & $>500$ & $>250$ & 500 & 500 & $>500$ & $>500$ & 87.07 \\
\hline & $48 h$ & $>500$ & 1.95 & $>500$ & $>500$ & $>250$ & $>500$ & $>500$ & $>500$ & $>500$ & 174.14 \\
\hline \multirow{2}{*}{$\mathbf{C G}$} & $24 h$ & $>500$ & 0.98 & 250 & $>500$ & $>250$ & 250 & 250 & $>500$ & $>500$ & 21.77 \\
\hline & $48 h$ & $>500$ & 1.95 & 500 & $>500$ & $>250$ & 500 & 500 & $>500$ & $>500$ & 69.65 \\
\hline \multirow{2}{*}{ TA } & $24 h$ & $>500$ & 1.95 & 500 & $>500$ & $>250$ & 500 & 500 & $>500$ & $>500$ & 4.35 \\
\hline & $48 h$ & $>500$ & 1.95 & $>500$ & $>500$ & $>250$ & $>500$ & $>500$ & $>500$ & $>500$ & 8.71 \\
\hline \multirow{2}{*}{$\mathbf{A F}$} & $24 h$ & $>500$ & $>500$ & $>500$ & $>500$ & $>250$ & $>500$ & $>500$ & $>500$ & $>500$ & $>419$ \\
\hline & $48 h$ & $>500$ & $>500$ & $>500$ & $>500$ & $>250$ & $>500$ & $>500$ & $>500$ & $>500$ & $>419$ \\
\hline \multirow{2}{*}{$\mathrm{AC}$} & $24 h$ & $>500$ & $>500$ & $>500$ & $>500$ & $>250$ & $>500$ & $>500$ & $>500$ & $>500$ & $>128$ \\
\hline & $48 \mathrm{~h}$ & $>500$ & $>500$ & $>500$ & $>500$ & $>250$ & $>500$ & $>500$ & $>500$ & $>500$ & $>128$ \\
\hline \multirow{2}{*}{ TM } & $72 \mathrm{~h}$ & $>500$ & $>500$ & $>500$ & $>500$ & $>250$ & $>500$ & $>500$ & $>500$ & $>500$ & 5.04 \\
\hline & $120 \mathrm{~h}$ & $>500$ & $>500$ & $>500$ & $>500$ & $>250$ & $>500$ & $>500$ & $>500$ & $>500$ & 8.00 \\
\hline
\end{tabular}

Unfortunately, the study has not included any simple (5-phenylalkylidene-4-oxo-2-thioxo-1,3thiazolidin-3-yl)acetic acid lacking phenylalkoxy substituents. Hence, it cannot be concluded whether this type of substitution is necessary for the PMT1-inhibitory activity. Regarding the rhodanineacetic part of the molecule, it was found that the free carboxylic group is needed since the analogues with an ester or amide group did not displayed activity in either the PMT1 or C. albicans assays.

\section{Conclusions}

A series of nine [(5Z)-(5-arylalkylidene-4-oxo-2-thioxo-1,3-thiazolidin-3-yl)]acetic acids was prepared. Four of the compounds have been reported previously, and five are novel compounds reported in the present paper for the first time. Antifungal properties of the studied compounds against a standard panel of eight fungal strains were tested. Only one compound, \{(5Z)-[4-oxo-5-(pyridin-2- 
ylmethylidene)-2-thioxo-1,3-thiazolidin-3-yl]\}acetic acid (2), inhibited the growth of Candida tropicalis 156, Candida krusei E 28, Candida glabrata 20/I and Trichosporon asahii 1188. The remaining substances were inactive. Further studies are required to elucidate the mechanism of antifungal effects of (5-arylalkylidene-4-oxo-2-thioxo-1,3-thiazolidin-3-yl)acetic acids and formulate structure-activity relationships.

\section{Experimental}

\subsection{General}

The synthesis of pyrazinecarbaldehyde and 5-substituted acetylpyrazines was described previously by Opletalova et al. [31,32,41]. Commercially available rhodanine- $N$-acetic acid (Fluka), benzaldehyde (VEB Laborchemie), pyridine-2-carbaldehyde (Aldrich), pyridine-3-carbaldehyde (Aldrich), pyridine-4-carbaldehyde (Aldrich), acetophenone (Reachim) were used for condensation. Pyrazinecarbonitrile (Fluka), pyrazinecarboxylic acid (Aldrich) were used as starting materials. For analysis, the samples of compounds were dried 24 hours in the dessicator at $1.33 \mathrm{kPa}$. The melting points were determined on a Boetius PHMK 05 (VEB Kombinat Nagema, Radebeul, Germany) and are uncorrected. Elemental analyses were performed with an EA 1110 CHNS Analyzer (Carlo Erba). The purity of the final compounds was checked by the HPLC separation module Waters Alliance 2695 XE (Waters Corp., Milford, MA, U.S.A.). The detection wavelength $210 \mathrm{~nm}$ was chosen. The solvent peaks in the (blank) chromatogram were deducted from the peaks in the chromatogram of the sample solution. The purity of individual compounds was determined from the area peaks in the chromatogram of the sample solution. UV spectra $(\lambda, \mathrm{nm})$ were determined on a Waters Photodiode Array Detector 2996 (Waters Corp., Milford, MA, U.S.A.) in ca. $6 \times 10^{-4} \mathrm{M}$ methanolic solution and $\log \varepsilon$ (the logarithm of molar absorption coefficient $\varepsilon$ ) was calculated for the absolute maximum $\lambda_{\max }$ of individual target compounds. Infrared spectra were recorded using $\mathrm{KBr}$ pellets on the spectrometer Nicolet Impact 400, for compounds 1-5, and on the FT-IR spectrometer Nicolet 6700, for compounds 6-9, (Nicolet - Thermo Scientific, USA). Wavenumbers are given in $\mathrm{cm}^{-1}$. All ${ }^{1} \mathrm{H}-\mathrm{NMR}$ and ${ }^{13} \mathrm{C}-\mathrm{NMR}$ spectra were recorded with a Varian Mercury-VxBB 300 spectrometer $\left(299.95 \mathrm{MHz}\right.$ for ${ }^{1} \mathrm{H}$ and 75.43 $\mathrm{MHz}$ for ${ }^{13} \mathrm{C}$; Varian Corp., Palo Alto, CA, USA). Chemical shifts were recorded as $\delta$ values in ppm and were indirectly referenced to tetramethylsilane (TMS) via the solvent signal $\left(2.49\right.$ for ${ }^{1} \mathrm{H}$, 39.7 for ${ }^{13} \mathrm{C}$ in DMSO- $d_{6}$ ).

\subsection{Synthesis}

A mixture of an aldehyde or a ketone $(0.009 \mathrm{~mol})$ and rhodanine-3-acetic acid (0.009 mol) was dissolved in glacial acetic acid and equivalent amount of acetanhydride and sodium acetate were added. Then the reaction mixture was refluxed for $3 \mathrm{~h}$. After cooling, the separated solid was filtered through a sintered filter, washed with distilled water $(50 \mathrm{~mL})$ and then with $50 \%$ ethanol $(50 \mathrm{~mL})$. The product was crystallized from glacial acetic acid.

[(5Z)-(5-Benzylidene-4-oxo-2-thioxo-1,3-thiazolidin-3-yl)]acetic acid (1). Yellow crystalline compound; Yield $66 \%$; Mp $247-248{ }^{\circ} \mathrm{C}\left(240{ }^{\circ} \mathrm{C}\right.$, acetic acid [3]); Anal. Calcd. for $\mathrm{C}_{12} \mathrm{H}_{9} \mathrm{NO}_{3} \mathrm{~S}_{2}$ 
(279.33): C 51.60\%, H 3.25\%, N 5.01\%, S 22.96\%; found: C 51.23\%, H 3.08\%, N 4.99\%, S 20.75\%; HPLC purity: 99.83\%; UV (nm), $\lambda_{\max } / \log \varepsilon: 375.6 / 3.28$; IR $\left(\mathrm{KBr}, \mathrm{cm}^{-1}\right): 3017(\mathrm{CH}), 1731(\mathrm{C}=\mathrm{O}(\mathrm{OH}))$, $1707(\mathrm{C}=\mathrm{O}), 759(\mathrm{C}=\mathrm{S})$; ${ }^{1} \mathrm{H}-\mathrm{NMR}\left(\mathrm{DMSO}-d_{6}\right), \delta: 7.89(1 \mathrm{H}, \mathrm{s}, \mathrm{CH}), 7.70-7.63$ (2H, m, H2', H6'), 7.60$7.51\left(3 \mathrm{H}, \mathrm{m}, \mathrm{H} 3^{\prime}, \mathrm{H}^{\prime}\right.$ ', H5'), $4.74\left(2 \mathrm{H}, \mathrm{s}, \mathrm{NCH}_{2}\right) ;{ }^{13} \mathrm{C}-\mathrm{NMR}$ (DMSO-d $\left.{ }_{6}\right), \delta: 193.5,167.5,166.6,134.2$, $133.0,131.4,131.0,129.8,122.0,45.2$.

\{(5Z)-[4-Oxo-5-(pyridin-2-ylmethylidene)-2-thioxo-1,3-thiazolidin-3-yl]\}acetic acid (2). Yellow crystalline compound; Yield 76\%; Mp 260-261 ${ }^{\circ} \mathrm{C}\left(253-254{ }^{\circ} \mathrm{C}\right.$, acetic acid [8]); Anal. Calcd. for $\mathrm{C}_{11} \mathrm{H}_{8} \mathrm{~N}_{2} \mathrm{O}_{3} \mathrm{~S}_{2}$ (280.32): C 47.13\%, H 2.88\%, N 9.99\%, S 22.88\%; found: C 46.75\%, H 3.12\%, N 9.81\%, S 22.59\%; HPLC purity: 98.95\%; UV (nm), $\lambda_{\max } / \log \varepsilon: 384.4 / 3.29 ; \mathrm{IR}\left(\mathrm{KBr}, \mathrm{cm}^{-1}\right): 3042$ $(\mathrm{CH}), 1740(\mathrm{C}=\mathrm{O}(\mathrm{OH})), 1705(\mathrm{C}=\mathrm{O}), 750(\mathrm{C}=\mathrm{S}) ;{ }^{1} \mathrm{H}-\mathrm{NMR}\left(\mathrm{DMSO}-d_{6}\right), \delta: 8.81(1 \mathrm{H}, \mathrm{d}, J=4.7 \mathrm{~Hz}$, H6), 8.03-7.92 (2H, m, H4, H5), $7.90(1 \mathrm{H}, \mathrm{s}, \mathrm{CH}), 7.50-7.43(1 \mathrm{H}, \mathrm{m}, \mathrm{H} 3), 4.73\left(2 \mathrm{H}, \mathrm{s}, \mathrm{NCH}_{2}\right) ;{ }^{13} \mathrm{C}-$ NMR (DMSO-d $\left.d_{6}\right), \delta: 199.9,167.6,166.7,151.2,149.8,138.0,129.7,128.7,126.4,124.6,44.8$.

\{(5Z)-[4-Oxo-5-(pyridin-3-ylmethylidene)-2-thioxo-1,3-thiazolidin-3-yl]\}acetic acid (3). Yellow crystalline compound; Yield 61\%; Mp 257-258 ${ }^{\circ} \mathrm{C}\left(261-264{ }^{\circ} \mathrm{C}\right.$, ethanol-acetic acid [27]); Anal. Calcd. for $\mathrm{C}_{11} \mathrm{H}_{8} \mathrm{~N}_{2} \mathrm{O}_{3} \mathrm{~S}_{2}$ (280.32): C 47.13\%, H 2.88\%, N 9.99\%, S 22.88\%; found C 46.89\%, H 3.15\%, N 9.86\%, S 23.17\%; HPLC purity: 98.75\%; UV (nm), $\lambda_{\max } / \log \varepsilon: 388.9 / 3.29 ; \mathrm{IR}\left(\mathrm{KBr}, \mathrm{cm}^{-1}\right)$ : $1704(\mathrm{C}=\mathrm{O}), 745(\mathrm{C}=\mathrm{S}) ;{ }^{1} \mathrm{H}-\mathrm{NMR}\left(\mathrm{DMSO}_{6}\right), \delta: 8.90(1 \mathrm{H}, \mathrm{d}, J=2.3 \mathrm{~Hz}, \mathrm{H} 2), 8.67(1 \mathrm{H}, \mathrm{d}$, $J=4.7 \mathrm{~Hz}, \mathrm{H} 6), 8.02(1 \mathrm{H}, \mathrm{d}, J=7.9 \mathrm{~Hz}, \mathrm{H} 4), 7.94(1 \mathrm{H}, \mathrm{s}, \mathrm{CH}), 7.59(1 \mathrm{H}, \mathrm{dd}, J=7.9 \mathrm{~Hz}, J=4.7 \mathrm{~Hz}$, H5), $4.75\left(2 \mathrm{H}, \mathrm{s}, \mathrm{NCH}_{2}\right) ;{ }^{13} \mathrm{C}-\mathrm{NMR}$ (DMSO-d $), \delta: 193.1,167.5,166.4,152.2,151.4,136.9,130.9$, $129.2,124.6,124.3,45.3$.

\{(5Z)-[4-Oxo-5-(pyridin-4-ylmethylidene)-2-thioxo-1,3-thiazolidin-3-yl]\}acetic acid (4). Yellow crystalline compound; Yield 80\%; Mp 277-280 ${ }^{\circ} \mathrm{C}\left(263-266^{\circ} \mathrm{C}\right.$, ethanol-acetic acid [27]); Anal. Calcd. for $\mathrm{C}_{11} \mathrm{H}_{8} \mathrm{~N}_{2} \mathrm{O}_{3} \mathrm{~S}_{2}$ (280.32): $\mathrm{C} 47.13 \%, \mathrm{H} 2.88 \%, \mathrm{~N} 9.99 \%, \mathrm{~S} 22.88 \%$; found: $\mathrm{C} 45.07 \%, \mathrm{H}$ $3.32 \%$, N 8.95\%, S 22.42\%; HPLC purity: 97.95\%; UV (nm), $\lambda_{\max } / \log \varepsilon: 389.1 / 3.29 ; \mathrm{IR}\left(\mathrm{KBr}, \mathrm{cm}^{-1}\right)$ : $3037(\mathrm{CH}), 1797(\mathrm{C}=\mathrm{O}(\mathrm{OH})), 1708(\mathrm{C}=\mathrm{O}), 754(\mathrm{C}=\mathrm{S}) ;{ }^{1} \mathrm{H}-\mathrm{NMR}\left(\mathrm{DMSO}-d_{6}\right), \delta: 8.78-8.72(2 \mathrm{H}, \mathrm{m}$, H2, H6), $7.86(1 \mathrm{H}, \mathrm{s}, \mathrm{CH}), 7.63-7.56(2 \mathrm{H}, \mathrm{m}, \mathrm{H} 3, \mathrm{H} 5), 4.75\left(2 \mathrm{H}, \mathrm{s}, \mathrm{NCH}_{2}\right) ;{ }^{13} \mathrm{C}-\mathrm{NMR}\left(\mathrm{DMSO}-d_{6}\right), \delta$ : 193.0, 167.4, 166.3, 151.0, $139.9130 .9,127.1,124.0,45.3$.

\{(5Z)-[4-Oxo-5-(pyrazin-2-ylmethylidene)-2-thioxo-1,3-thiazolidin-3-yl]\}acetic acid (5). Yellow crystalline compound; Yield 71\%; Mp 266-268 ${ }^{\circ} \mathrm{C}$; Anal. Calcd. for $\mathrm{C}_{10} \mathrm{H}_{7} \mathrm{~N}_{3} \mathrm{O}_{3} \mathrm{~S}_{2}$ (281.31): C 42.70\%, H 2.51\%, N 14.94\%, S 22.80\%; found: C 42.57\%, H 2.83\%, N 14.80\%, S 25.46\%; HPLC purity: 97.85\%; UV (nm), $\lambda_{\max } / \log \varepsilon: 389.2 / 3.29$; IR $\left(\mathrm{KBr}, \mathrm{cm}^{-1}\right): 3051(\mathrm{CH}), 1737(\mathrm{C}=\mathrm{O}(\mathrm{OH})), 1709(\mathrm{C}=\mathrm{O})$, $740(\mathrm{C}=\mathrm{S}) ;{ }^{1} \mathrm{H}-\mathrm{NMR}\left(\mathrm{DMSO}-d_{6}\right), \delta: 9.14(1 \mathrm{H}, \mathrm{s}, \mathrm{H} 3), 8.87(1 \mathrm{H}, \mathrm{s}, \mathrm{H} 5), 8.67(1 \mathrm{H}, \mathrm{s}, \mathrm{H} 6), 7.94(1 \mathrm{H}, \mathrm{s}$, $\mathrm{CH}), 4.57\left(2 \mathrm{H}, \mathrm{s}, \mathrm{NCH}_{2}\right) ;{ }^{13} \mathrm{C}-\mathrm{NMR}\left(\mathrm{DMSO}_{-}\right), \delta: 198.4,167.4,166.7,148.8,147.4,144.7,129.1$, $125.6,46.2$.

[(5Z)-\{4-Oxo-5-[1-(pyrazin-2-ylethylidene)]-2-thioxo-1,3-thiazolidin-3-yl\}] acetic acid (6). Dark orange solid; Yield 75\%; Mp 261-263 ${ }^{\circ} \mathrm{C}$; Anal. Calcd. for $\mathrm{C}_{11} \mathrm{H}_{9} \mathrm{~N}_{3} \mathrm{O}_{3} \mathrm{~S}_{2}$ (295.34): C 44.74\%, $\mathrm{H}$ $3.07 \%$, N 14.23\%, S 21.71\%; found: C 44.41\%, H 3.20\%, N 14.05\%, S $21.16 \%$; HPLC purity: 
98.95\%; UV (nm), $\lambda_{\max } / \log \varepsilon: 388.0 / 3.19 ; \mathrm{IR}\left(\mathrm{KBr}, \mathrm{cm}^{-1}\right): 3412(\mathrm{C}-\mathrm{OH}), 3042(\mathrm{CH}), 1716(\mathrm{C}=\mathrm{O}), 784$ $(\mathrm{C}=\mathrm{S}) ;{ }^{1} \mathrm{H}-\mathrm{NMR}$ (DMSO-d $\left.{ }_{6}\right), \delta: 3.37$ (1H, bs, OH), 9.33 (1H, d, J=1.7 Hz, H3), 8.92-8.89 (1H, m, H5), $8.74(1 \mathrm{H}, \mathrm{d}, J=2.5 \mathrm{~Hz}, \mathrm{H} 6), 4.71\left(2 \mathrm{H}, \mathrm{s}, \mathrm{NCH}_{2}\right), 2.92\left(3 \mathrm{H}, \mathrm{s}, \mathrm{CH}_{3}\right) ;{ }^{13} \mathrm{C}-\mathrm{NMR}\left(\mathrm{DMSO}-d_{6}\right), \delta: 199.5$, $167.7,166.5,149.4,145.7,144.7,142.3,139.5,123.2,44.7,15.5$.

[(5Z)-\{5-[1-(5-Butylpyrazin-2-yl)ethylidene]-4-oxo-2-thioxo-1,3-thiazolidine-3-yl\}]acetic acid

Yellow crystalline compound; Yield 77\%; Mp 213-215 ${ }^{\circ} \mathrm{C}$; Anal. Calcd. for $\mathrm{C}_{15} \mathrm{H}_{17} \mathrm{~N}_{3} \mathrm{O}_{3} \mathrm{~S}_{2}$ (351.45): C $51.26 \%, \mathrm{H} 4.88 \%$, N $11.96 \%$, S 18.25\%; found: $\mathrm{C} \quad 50.84 \%, \mathrm{H} \quad 4.89 \%, \mathrm{~N} 11.96 \%$, S 17.88\%; HPLC purity: 99.03\%; UV (nm), $\lambda_{\max } / \log \varepsilon: 388.0 / 3.29 ; \mathrm{IR}\left(\mathrm{KBr}, \mathrm{cm}^{-1}\right): 3398(\mathrm{C}-\mathrm{OH})$, $1706(\mathrm{C}=\mathrm{O}), 758(\mathrm{C}=\mathrm{S}) ;{ }^{1} \mathrm{H}-\mathrm{NMR}\left(\mathrm{DMSO}_{6}\right), \delta: 9.22(1 \mathrm{H}, \mathrm{d}, J=1.4 \mathrm{~Hz}, \mathrm{H} 3), 8.81(1 \mathrm{H}, \mathrm{d}$, $J=1.4 \mathrm{~Hz}, \mathrm{H} 6), 4.71\left(2 \mathrm{H}, \mathrm{s}, \mathrm{NCH}_{2}\right), 2.90\left(3 \mathrm{H}, \mathrm{s}, \mathrm{CH}_{3}\right), 2.86\left(2 \mathrm{H}, \mathrm{t}, J=7.5 \mathrm{~Hz}, \mathrm{CH}_{2}\right), 1.78-1.63(2 \mathrm{H}$, $\left.\mathrm{m}, \mathrm{CH}_{2}\right), 1.41-1.25\left(2 \mathrm{H}, \mathrm{m}, \mathrm{CH}_{2}\right), 0.90\left(3 \mathrm{H}, \mathrm{t}, J=7.5 \mathrm{~Hz}, \mathrm{CH}_{3}\right) ;{ }^{13} \mathrm{C}-\mathrm{NMR}\left(\mathrm{DMSO}-d_{6}\right), \delta: 199.5$, $167.7,166.5,157.7,146.8,144.8,141.7,139.9,122.0,44.7,34.4,30.8,22.0,15.5,13.9$.

[(5Z)-\{5-[1-(5-tert-Butylpyrazin-2-yl)ethylidene]-4-oxo-2-thioxo-1,3-thiazolidin-3-yl\}] acetic acid (8). Yellow crystalline compound; Yield 63\%; Mp 254-261 ${ }^{\circ} \mathrm{C}$ (decomp.); Anal. Calcd. for $\mathrm{C}_{15} \mathrm{H}_{17} \mathrm{~N}_{3} \mathrm{O}_{3} \mathrm{~S}_{2}$ (351.44): C 51.26\%, H 4.88\%, N 11.96\%, S 18.25\%; found: C $50.85 \%, \mathrm{H} 5.10 \%$, N 12.12\%, S 18.32\%; HPLC purity: 98.42\%; UV (nm), $\lambda_{\max } / \log \varepsilon: 388.0 / 3.16$; IR (KBr, cm $\left.{ }^{-1}\right): 3392$ $(\mathrm{C}-\mathrm{OH}), 1703(\mathrm{C}=\mathrm{O}), 758(\mathrm{C}=\mathrm{S}) ;{ }^{1} \mathrm{H}-\mathrm{NMR}\left(\mathrm{DMSO}-d_{6}\right), \delta: 13.37(1 \mathrm{H}, \mathrm{bs}, \mathrm{OH}), 9.26(1 \mathrm{H}, \mathrm{d}, J=1.4$ $\mathrm{Hz}, \mathrm{H} 3), 9.03(1 \mathrm{H}, \mathrm{d}, J=1.4 \mathrm{~Hz}, \mathrm{H} 6), 4.71\left(2 \mathrm{H}, \mathrm{s}, \mathrm{NCH}_{2}\right), 2.92\left(3 \mathrm{H}, \mathrm{s}, \mathrm{CH}_{3}\right), 1.39\left(9 \mathrm{H}, \mathrm{s}, \mathrm{CH}_{3}\right) ;{ }^{13} \mathrm{C}-$ NMR (DMSO- $\left.d_{6}\right), \delta: 199.6,167.8,166.5,163.9,146.5,144.1,139.7,139.1,122.2,44.7,36.9,29.6$, 15.5 .

[(5Z)-\{5-[1-(5-Benzylpyrazin-2-yl)ethylidene]-4-oxo-2-thioxo-1,3-thiazolidin-3-yl\}]acetic acid (9). Yellow crystalline compound; Yield 31\%; Mp 224-225 ${ }^{\circ} \mathrm{C}$; Anal. Calcd. for $\mathrm{C}_{18} \mathrm{H}_{15} \mathrm{~N}_{3} \mathrm{O}_{3} \mathrm{~S}_{2}$ (385.47): C $56.09 \%, \mathrm{H} 3.92 \%, \mathrm{~N} 10.90 \%$, S 16.64\%; found: $\mathrm{C} \quad 55.29 \%, \mathrm{H} \quad 3.96 \%, \mathrm{~N} \quad 10.86 \%$, S 16.46\%; HPLC purity: 99.62\%; UV (nm), $\lambda_{\max } / \log \varepsilon: 386.8 / 3.32 ; \mathrm{IR}\left(\mathrm{KBr}, \mathrm{cm}^{-1}\right): 3436(\mathrm{C}-\mathrm{OH})$, $1729(\mathrm{C}=\mathrm{O}(\mathrm{OH})), 1697(\mathrm{C}=\mathrm{O}), 754(\mathrm{C}=\mathrm{S}) ;{ }^{1} \mathrm{H}-\mathrm{NMR}\left(\mathrm{DMSO}-d_{6}\right), \delta: 9.22(1 \mathrm{H}, \mathrm{d}, J=1.4 \mathrm{~Hz}, \mathrm{H} 3), 8.88$ $(1 \mathrm{H}, \mathrm{d}, J=1.4 \mathrm{~Hz}, \mathrm{H} 6), 7.36-7.18(5 \mathrm{H}, \mathrm{m}, \mathrm{Ar}), 4.70\left(2 \mathrm{H}, \mathrm{s}, \mathrm{NCH}_{2}\right), 4.23\left(2 \mathrm{H}, \mathrm{s}, \mathrm{CH}_{2}\right), 2.89(3 \mathrm{H}, \mathrm{s}$, $\mathrm{CH}_{3}$ ); ${ }^{13} \mathrm{C}-\mathrm{NMR}$ (DMSO- $d_{6}$ ), $\delta: 199.4,167.7,166.5,156.3,147.1,145.0,141.8,139.7,138.5,129.3$, $128.8,126.8,122.3,44.8,40.9,15.5$.

\subsection{Lipophilicity HPLC determination (capacity factor $k / c a l c u l a t e d ~ l o g ~ k$ )}

The HPLC separation module Waters Alliance 2695 XE and Waters Photodiode Array Detector 2996 (Waters Corp., Milford, MA, U.S.A.) were used. The chromatographic column Symmetry ${ }^{\circledR}$ $\mathrm{C}_{18} 5 \mu \mathrm{m}, 4.6 \times 250 \mathrm{~mm}$, Part No. WAT054275, (Waters Corp.) was used. The HPLC separation process was monitored by Millennium32 ${ }^{\circledR}$ Chromatography Manager Software, Waters 2004 (Waters Corp.). A mixture of $\mathrm{MeOH}$ p.a. (70.0\%) and $\mathrm{H}_{2} \mathrm{O}-\mathrm{HPLC}$ - Mili-Q Grade (30.0\%) was used as a mobile phase. The total flow of the column was $0.9 \mathrm{~mL} / \mathrm{min}$, injection $30 \mu \mathrm{L}$, column temperature $30{ }^{\circ} \mathrm{C}$ and sample temperature $10{ }^{\circ} \mathrm{C}$. The detection wavelength $210 \mathrm{~nm}$ was chosen. The KI 
methanolic solution was used for the dead time $\left(t_{D}\right)$ determination. Retention times $\left(t_{R}\right)$ were measured in minutes.

The capacity factors $k$ were calculated using the Millennium32 ${ }^{\circledR}$ Chromatography Manager Software according to formula $k=\left(t_{R}-t_{D}\right) / t_{D}$, where $t_{R}$ is the retention time of the solute, whereas $t_{D}$ denotes the dead time obtained via an unretained analyte. $\log k$, calculated from the capacity factor $k$, is used as the lipophilicity index converted to $\log P$ scale. The $\log k$ values of the individual compounds are shown in Table 1.

\subsection{In vitro evaluation of antifungal activity}

All strains were subcultured on Sabouraud dextrose agar (SDA, Difco) and maintained on the same medium at $4{ }^{\circ} \mathrm{C}$. Prior to testing, each strain was passaged onto SDA and fungal inocula were prepared by suspending yeasts or conidia or sporangiospores in sterile $0.85 \%$ saline. The cell density was adjusted, using the Bürker's chamber, to yield a stock suspension of $(1.0 \pm 0.2) \times 105 \mathrm{CFU} / \mathrm{mL}$. The final inoculum was made by 1:20 dilution of the stock suspension with the test medium. The compounds were dissolved in dimethyl sulfoxide (DMSO) and antifungal activity was determined in the tissue culture medium RPMI 1640 (Sevapharma, Prague, Czech Republic) buffered to pH 7.0 with 0.165 M 3-morpholinopropane-1-sulfonic acid (Sigma-Aldrich). Controls consisted of medium and DMSO alone. The final concentration of DMSO in the test medium did not exceed $1 \%(\mathrm{v} / \mathrm{v})$ of the total solution composition. The minimum inhibitory concentration (MIC), defined as $80 \%$ inhibition of fungal growth compared to control, were determined after 24 and $48 \mathrm{~h}$ of static incubation at $35^{\circ} \mathrm{C}$. In the case of T. mentagrophytes the MICs were recorded after 72 and $120 \mathrm{~h}$. Fluconazole (Pfizer, New York, NY, USA) was used as reference antifungal drugs. The antifungal evaluation results are showed in Table 2.

\section{Acknowledgements}

The study was supported by the Ministry of Education, Youth and Sports (Research Project MSM 0021620822).

\section{References}

1. Boyd, D.B. On the rhodanines and their presence in biologically active ligands. J. Mol. Struct. Theochem. 1997, 401, 227-234.

2. Körner, H. Derivatives of dithiocarbaminoacetic acid. Ber. Dtsch. Chem. Ges. 1908, 41, 1901-1905; [Chem. Abstr. 1908, 2, 12300].

3. Andreasch, R. Substituted rhodaninic acids and their aldehyde condensation products. VII. Monatsh. Chem. 1908, 29, 399-419; [Chem. Abstr. 1908, 2, 14948].

4. Taniyama, H.; Yasui, B.; Takehara; N.; Uchida, H. Chemotherapeutics for Mycobacterium tuberculosis. XIX. Synthesis and antibacterial activity of some 3-substituted rhodanines. Yakugaku Zasshi 1959, 79, 1465-1468; [Chem. Abstr. 1960, 54, 34220].

5. Singh, J.; Nathan, C.F.; Bryk, R.; Samy, R.; Pupek, K.; Gurney, M. Cyclic carboxylic acid rhodanine derivatives for the treatment and prevention of tuberculosis. WO Pat. 2008005651, 2008 . 
6. Allan, F.J.; Allan, G.G.; Crank, G.; Jack, J. The condensation of rhodanine and derivatives with benzaldehyde sulphonic acids. Recl. Trav. Chim. Pays Bas 1960, 79, 247-254.

7. Allan, F.J.; Allan, G.G. The condensation of rhodanine and derivatives with 4-antipyrinaldehyde. Can. J. Chem. 1961, 39, 1397-1399.

8. Allan, F.J.; Allan, G.G.; Thompson, J.B. The condensation of rhodanine and derivatives with pyridine and quinoline aldehydes. Recl. Trav. Chim. Pays Bas 1961, 80, 403-408.

9. Allan, F.J.; Allan, G.G. The condensation of rhodanine and derivatives with some indole aldehydes. Monatsh. Chem. 1963, 94, 1434-4475; [Chem. Abstr. 1963, 59, 48312].

10. Allan, F.J.; Allan, G.G. The condensation of rhodanine and derivatives with aromatic aldehydes containing iodine. Recl. Trav. Chim. Pays Bas 1962, 82, 177-181.

11. Allan, F.J.; Allan, G.G. Izoxazolylmethylenerhodanines. Recl. Trav. Chim. Pays Bas 1964, 83, 1299-1300.

12. Orchard, M.G.; Neuss, J.C.; Galley, C.M.S. Benzylidene thiazolidinediones and their use as antimycotic agents. WO Pat. 02017915, 2002.

13. Orchard, M.G.; Neuss, J.D. Preparation of thiazolidines as antifungal agents. WO Pat. 0222612, 2002.

14. Orchard, M.G. Preparation of 5-benzylidene-4-thiazolidinone-2-thione antifungal agents. WO Pat. 03070238, 2003.

15. Orchard, M.G.; Neuss, J.C.; Galley, C.M.S.; Carr, A.; Porter, D.W.; Smith, P.; Scopes, D.I.C.; Haydon, D.; Vousden, K.; Stubberfield, C.R.; Young, K.; Page, M. Rhodanine-3-acetic acid derivatives as inhibitors of fungal protein mannosyl transferase 1 (PMT 1). Bioorg. Med. Chem. Lett. 2004, 14, 3975-3978.

16. Dovlatyan, V.V.; Avetisyan, F.V. 2-Thioxo-4-imino(oxo)-1,3-thiazolidin-3-ylacetic acid and its alkyl esters. Armyanskii Khim. Zh. 1973, 26, 494-498; [Chem. Abstr. 1973, 79, 115482].

17. Inamori, Y.; Muro, C.; Tanaka, R.; Adachi, A.; Miyamoto, K.; Tsujibo, H. Phytogrowthinhibitory activity of sulfur-containing compounds. I. Inhibitory activities of thiazolidine derivatives on plant growth. Chem. Pharm. Bull. 1992, 40, 2854-2856.

18. Muro, C.; Yasuda, M.; Sakagami, Y.; Yamada, T.; Tsujibo, H.; Numata, A.; Inamori, Y. Inhibitory activities of rhodanine derivatives on plant growth. Biocsi. Biotechnol. Biochem. 1996, 60, 1368-1371.

19. Frankov, I.A.; Kirillov, M.V.; Sokolova, T.N.; Skupskaya, R.; Kharitonovich, A.N.; Chizhevskaya, I.I. Synthesis and pharmacological properties of alkyl derivatives of 3-carboxyalkylrhodanine. Khim.-Farm. Zh. 1985, 19, 943-946; [Chem. Abstr. 1986, 104, 129831].

20. Friebe, W.G.; Krell, H.W.; Woelle, S.; Wolff, H.P. Thiazolidine carboxylic acid derivatives and their use in the treatment of cancer. WO Pat. 0157006, 2001.

21. Singh, R.; Ramesh, U.V.; Goff, D.; Laidig, G.; Issakani, S.D.; Huang, J.; Payan, D.G.; Clough, J. Rhodanine derivatives and pharmaceutical compositions containing them. WO Pat. 2004043955 , 2004.

22. Esswein, A.; Schaefer, W.; Tsaklakidis, C.; Honold, K.; Kaluza, K. Rhodanine carboxylic acid derivatives for the treatment and prevention of metabolic bone disorders. US Pat. App. 2003032813, 2003.

23. Esswein, A.; Schaefer, W.; Tsaklakidis, C.; Honold, K.; Kaluza, K. Rhodanine carboxylic acid derivatives for the treatment and prevention of metabolic bone disorders. US Pat. App. 6673816, 2004. 
24. Smith, T.J.; Young, B.L.; Denton, H.; Hughes, D.L.; Wagner, G.W. First small molecular inhibitors of T. brucei dolicholphospate mannose synthase (DPMS), a validated target in African sleeping sickness. Bioorg. Med. Chem. Lett. 2009, 19, 1749-1752.

25. Ziegler, D. Treatment of diabetic neuropathy and neuropathic pain: how far have we come? Diabetes Care 2008, 31 (Suppl. 2), S255-S261.

26. Ramirez, M.A.; Borja, N.L. Epalrestat: An aldose reductase inhibitor for the treatment of diabetic neuropathy. Pharmacotherapy 2008, 28, 646-655.

27. Tanaouchi, T.; Kawamura, M.; Ajima, A.; Mohri, T.; Hayashi, M.; Terashima, H.; Hirata, F.; Morimura, T. Rhodanine derivatives, process for their preparation, and aldose reductase inhibitor containing the rhodanine derivatives as active ingredient. EP Pat. 0047109, 1982.

28. Tanaouchi, T.; Kawamura, M.; Ajima, A.; Mohri, T.; Hayashi, M.; Terashima, H.; Hirata, F.; Morimura, T. Rhodanine derivatives, process for their preparation, and aldose reductase inhibitor containing the rhodanine derivatives as active ingredient. US Pat. 4464382, 1984.

29. Tomasic, T.; Masic, L.P. Rhodanine as a privileged scaffold in drug discovery. Curr. Med. Chem. 2009, 16, 1596-1629.

30. Lipinski, C.A.; Lombardo, F.; Dominy, B.W.; Feeney, P.J. Experimental and computational approaches to estimate solubility and permeability in drug discovery and development settings. Adv. Drug Delivery Rev. 1997, 23, 3-25.

31. Avdeef, A. Physicochemical profiling (permeability, solubility, charge state). Curr. Topics Med. Chem. 2001, 1, 277-351.

32. Pliska, V. Lipophilicity in drug action and toxicology. In Methods and Principles in Medicinal Chemistry, 1st ed.; Pliska, V., Testa, B., van der Waterbeemd, H., Eds.; Wiley-VCH: Weinheim, Germany, 1996; Vol. 4, pp. 1-6.

33. Gocan, S.; Cimpan, G.; Comer, J. Lipophilicity measurements by liquid chromatography. Adv. Chromatogr. 2006, 44, 79-176.

34. Opletalova, V.; Dolezal, M.; Hartl, J. Oxidation of hydroxymethylpyrazine using sonication. In Book of Abstracts of the 23rd Conference Drug Synthesis and Analysis; In the 23rd Conference Drug Synthesis and Analysis, Bratislava, Slovakia, October 4-10, 1994; p. 25.

35. Opletalova, V.; Patel, A.; Boulton, M.; Dundrova, A.; Lacinova, E.; Prevorova, M.; Appeltauerova, M.; Coufalova, M. 5-Alkyl-2-pyrazinecarboxamides, 5-alkyl-2pyrazinecarbonitriles and 5-alkyl-2-acetylpyrazines as synthetic intermediates for antiinflammatory agents. Collect. Czech Chem. Commun. 1996, 61, 1093-1101.

36. Opletalova, V.; Jampilek, J.; Chlupacova, M.; Dolezel, J.; Dohnal, J. Chromatographic and computational study of hydrophobic properties of ring substituted pyrazinecarbonitriles and acetylpyrazines. In Proceedings of the 9th International Electronic Conference on Synthetic Organic Chemistry (ECSOC-9), November 1-30, 2005 [CD-ROM ed.]; Seijas, J.A., Tato, M.P. V., Eds.; MDPI: Basel, Switzerland, 2005; c004.

37. Jampilek, J.; Opletalova, V.; Grafnetterova, T.; Dohnal, J. Thiosemicarbazones of acetylpyrazines: preparation and their hydrophobic properties. In Proceedings of the 9th International Electronic Conference on Synthetic Organic Chemistry (ECSOC-9), November 130, 2005 [CD-ROM ed.]; Seijas, J.A., Tato, M.P.V., Eds.; MDPI: Basel, Switzerland, 2005; a001.

38. Jampilek, J.; Opletalova, V.; Dohnal, J. N,N-Dimethylthiosemicarbazones of acetylpyrazines: Preparation and their hydrophobic properties. In Proceedings of the 10th International Electronic 
Conference on Synthetic Organic Chemistry (ECSOC-10), November 1-30, 2006 [CD-ROM ed.]; Seijas, J. A., Tato, M. P. V., Eds.; MDPI: Basel, Switzerland, 2006; a004.

39. Jampilek, J.; Dolezal, M.; Osicka, Z.; Kunes, J.; Kralova, K. Substituted pyrazine-2carboxamides: preparation and their photosynthesis-inhibiting activity. In Proceedings of the 7th International Electronic Conference on Synthetic Organic Chemistry (ECSOC-7), November 130, 2003 [CD-ROM ed.]; Seijas, J.A., Tato, M.P.V., Eds.; MDPI: Basel, Switzerland, 2003; c001.

40. Chlupacova, M.; Opletalova, V.; Kunes, J.; Silva, L.; Buchta, V.; Duskova, L.; Kralova, K. Synthesis and biological evaluation of some ring-substituted (E)-3-aryl-1-pyrazin-2-ylprop-2-en1-ones. Folia Pharm. Univ. Carol. 2005, 33, 31-43.

41. Opletalova, V.; Pour, M.; Kunes, J.; Buchta, V.; Silva, L.; Kralova, K.; Chlupacova, M.; Meltrova, D.; Peterka, M.; Poslednikova, M. Synthesis and biological evaluation of (E)-3(nitrophenyl)-1-(pyrazin-2-yl)prop-2-en-1-ones. Collect. Czech Chem. Commun. 2006, 71, 44-58.

42. Dolezal, M.; Palek, L.; Vinsova, J.; Buchta, V.; Jampilek, J.; Kralova, K. Substituted pyrazinecarboxamides. Synthesis and biological evaluation. Molecules 2006, 11, 242-256.

43. Jampilek, J.; Opletalova, V.; Dolezel, J.; Dohnal, J. Preparation and hydrophobic properties of 5-arylalkylidenerhodanines. In Proceedings of the 11th International Electronic Conference on Synthetic Organic Chemistry (ECSOC-11), November 1-30, 2007 [CD-ROM ed.]; Seijas, J.A., Tato, M.P.V., Eds.; MDPI: Basel, Switzerland, 2003; a012.

44. Kucerova-Chlupacova, M.; Opletalova, V.; Jampilek, J.; Dolezel, J.; Dohnal, J.; Kunes, J.; Pour, M.; Kunes, J.; Vorisek, V. New hydrophobicity constants of substituents in pyrazine rings derived from RP-HPLC Study. Collect. Czech Chem. Comm. 2008, 73, 1-18.

45. Dolezel, J.; Hirsova, P.; Opletalova, V.; Vejsova, M.; Jampilek, J. Synthesis and biological evaluation of rhodanine and $N$-substituted rhodanine derivatives. Book of Abstracts of the 37th Conference Drug Synthesis and Analysis, Brno, Czech Republic, September 8-10, 2008; Chem. Listy 2008, 102, S215.

46. Opletalova, V.; Kalinowski, D.; Vejsova, M.; Kunes, J.; Pour, M., Jampilek, J.; Buchta, V.; Richardson, D.R. Identification and characterization of thiosemicarbazones with anti-fungal and anti-tumor effects: Cellular iron-chelation mediating cytotoxic activity. Chem. Res. Toxicol. 2008, $21,1878-1889$.

47. Zhou, J.F.; Song, Y.Z.; Zhu, F.X.; Zhu, Y.L. Facile synthesis of 5-benzylidene rhodanine derivatives under microwave irradiation. Synthetic Commun. 2006, 36, 3297-3303.

48. Sing, W.T.; Lee, C.L.; Yeo, S.L.; Lim, S.P.; Sim, M.M. Arylalkylidene rhodanine with bulky and hydrophobic functional group as selective HCV NS3 protease inhibitor. Bioorg. Med. Chem. Lett. 2001, 11, 91-94.

49. Khodair, A.I. A convenient synthesis of 2-arylidene-5H-thiazolo[2,3-b]quinazoline-3,5[2H]diones and their benzoquinazoline derivatives. J. Heterocycl. Chem. 2002, 39, 1153-1160.

50. Ohishi, Y.; Mukai, T.; Nagahara, M.; Yajima, M.; Kajikawa, N.; Miahara, K.; Takano, T. Preparations of 5-alkylmethylidene-3-carboxymethylrhodanine derivatives and their aldose reductase inhibitory activity. Chem. Pharm. Bull. 1990, 38, 1911-1919.

51. Whitessit, C.A.; Simon, R.L.; Reel, J.K.; Sigmund, S.K.; Phillips, M.L.; Shadle, J.K.; Heintz, L.W.; Koppel, G.A.; Hunden, D.C.; Lifer, S.L.; Berry, D.; Ray, J.; Little, S.P.; Liu, X.; Marshall, W.S.; Panetta, J.A. Synthesis and structure-activity relationships of benzophenones as inhibitors of cathepsin D. Bioorg. Med. Chem. Lett. 1996, 6, 2157-2162. 
52. Kerns, E.H.; Li, D. Drug-Like Properties: Concept, Structure Design and Methods; Elsevier: San Diego, CA, USA, 2008.

53. Valko, K. Application of high-performance liquid chromatography based measurements of lipophilicity to model biological distribution. J. Chromatogr. A 2004, 1037, 299-310.

54. Valko, K.; Du, C.M.; Bevan, C.; Reynolds, D.P.; Abraham, M.H. Rapid method for the estimation of octanol/water partition coefficient $\left(\log P_{\text {oct }}\right)$ from gradient RP-HPLC retention and a hydrogen bond acidity term $\left(\mathrm{Sa}_{2} \mathrm{H}\right)$. Curr. Med. Chem. 2001, 8, 1137-1146.

55. Cimpan, G.; Irimie, F.; Gocan, S.; Claessens, H.A. Role of stationary phase and eluent composition on the determination of $\log P$ values of $N$-hydroxyethylamide of aryloxyalkylen and pyridine carboxylic acids by reversed-phase high-performance liquid chromatography. J. Chromatogr. B 1998, 714, 247-261.

56. Hartmann, T.; Schmitt, J. Lipophilicity-beyond octanol/water: A short comparison of modern technologies. Drug Discov. Today Technol. 2004, 1, 431-439.

57. Nasal, A.; Siluk, D.; Kaliszan, R. Chromatographic retention parameters in medicinal chemistry and molecular pharmacology. Curr. Med. Chem. 2003, 10, 381-426.

58. Piraprez, G.; Herent, M.F.; Collin, S. Determination of the lipophilicity of aroma compounds by RP-HPLC. Flavour Fragr. J. 1998, 13, 400-408.

59. Yamagami, C.; Iwasaki, K.; Ishikawa, A. Hydrophobicity parameters determined by reversedphase liquid chromatography. XII. Comparison of capacity factors and octane/methanol-water partition coefficients for monosubstituted pyrazines, and effect of octanol added to both partitioning systems. Chem. Pharm. Bull. 1997, 45, 1653-1658.

60. Yamagami, C.; Araki, K.; Ohnishi, K.; Hanasato, K.; Inaba, H.; Aono, M.; Ohta, A. Measurement and prediction of hydrophobicity parameters for highly lipophilic compounds: Application of the HPLC column-switching technique to measurement of $\log P$ of diarylpyrazines. J. Pharm. Sci. 1999, 88, 1299-1304.

61. Yamagami, C.; Kawase, K.; Iwaki, K. Hydrophobicity parameters determined by reversed-phase liquid chromatography. XV: Optimal conditions for prediction of $\log P_{\text {oct }}$ by using RP-HPLC procedures. Chem. Pharm. Bull. 2002, 50, 1578-1583.

62. Sortino, M.; Delgado, P.; Juarez, S.; Quiroga, J.; Abonía, R.; Insuasty, B.; Nogueras, M.; Roredo, L.; Garibotto, F. M.; Enriz, R.D.; Zacchino, S. A. Synthesis and antifungal activity of (Z)-5arylidenerhodanines. Bioorg. Med. Chem. 2007, 15, 484- 494.

63. Petrik, P.; Kunes, J.; Vejsova, M.; Jampilek, J.; Spaningerova, E.; Kesetovicova, D.; Vlckova, M.; Majd, M.; Kalafutova, S.; Opletalova, V. Antifungal and antimycobacterial properties of 5-arylmethylidenerhodanines and their $N^{3}$-substituted analogues. Book of Abstracts of the 5th International Postgraduate Research Symposium on Pharmaceutics (IPORSIP-2007), Istanbul, September 13-15, 2007. Acta Pharma. Sci. 2007, 49 (Suppl.), 77.

Sample Availability: Samples of the compounds are available from the authors.

(C) 2009 by the authors; licensee Molecular Diversity Preservation International, Basel, Switzerland. This article is an open-access article distributed under the terms and conditions of the Creative Commons Attribution license (http://creativecommons.org/licenses/by/3.0/). 